\title{
12 Lead Placement Unspecified
}

National Cancer Institute

\section{Source}

National Cancer Institute. 12 Lead Placement Unspecified. NCI Thesaurus. Code C71101.

An electrocardiogram (ECG) lead placement whereby 12 leadpoints are recorded but the position of the leads is unspecified. 